\title{
Uso de recursos de salud por parte de pacientes adultos con cardiopatía congénita en Chile
}

\author{
Fernando Baraona ${ }^{1-2}$, Felipe Morán ${ }^{3}$, Julieth Huenur ${ }^{3}$, Felipe Moya ${ }^{3}$, Rodrigo González ${ }^{1-2}$, \\ Loreto Sore $^{2}$, Ana Rubilar ${ }^{2}$, Polentzi Uriarte. ${ }^{2}$ \\ 1 División de Enfermedades Cardiovasculares, Facultad de Medicina, Pontificia Universidad \\ Católica de Chile. \\ 2 Centro de Cardiopatías Congénitas del Adulto, Instituto Nacional del Tórax, Santiago, Chile. \\ 3 Escuela de Medicina, Facultad de Medicina, Pontificia Universidad Católica de Chile. \\ * Sin fuentes de financiamiento que declarar.
}

Introducción: Reportes internacionales han descrito el mayor uso de recursos de salud por parte de los pacientes adultos con cardiopatía congénita (CC).

Objetivo: Describir el uso de recursos de salud por parte de esta población en términos de frecuencia de controles médicos, consultas a servicio de urgencia, hospitalizaciones y procedimientos realizados. También se buscó explorar la frecuencia de control odontológico y la indicación de profilaxis de endocarditis como una medida de calidad de atención.

Metodología: Se realizó un estudio cuantitativo de corte transversal, analítico e individual en pacientes controlados en el Instituto Nacional del Tórax, durante el período 2014-2015. Los pacientes respondieron una encuesta en forma autónoma. Se realizó análisis por grupos según sexo y tipo de CC según el consenso de Bethesda (simple, moderada o compleja). El análisis estadístico se realizó a través del programa SPSS.

Resultados: Se recolectaron 363 encuestas válidas que correspondieron a 180 hombres $(49,6 \%)$ y con una edad promedio de 29,2 $\pm 13,3$ años. Según el tipo de CC: simple 78 pacientes $(21,5 \%)$, moderada $161(44,4 \%)$ y compleja $124(34,2 \%) .296$ pacientes $(84,6 \%)$ tuvieron el último control médico hace menos de un año. 151 pacientes $(41,6 \%)$ han consultado una o más veces al servicio de urgencia. 76 pacientes $(20,9 \%)$ han sido hospitalizados una o más veces y 54 pacientes $(14,9 \%)$ se han realizado uno o más procedimientos en el último año. En el análisis por tipo de CC no se encontraron diferencias significativas en ninguno de los parámetros evaluados. En el análisis según sexo, se observó que las mujeres consultan más al servicio de urgencia que los hombres $(48,1 \%$ vs $35 \%, p<0,05)$. En cuanto a salud oral, 122 pacientes $(33,6 \%)$ no ha realizado un control odontológico hace más de 1 año y de aquellos pacientes que asistieron al dentista, 165 de ellos $(45,5 \%)$ no recibió profilaxis antibiótica para endocarditis en su última atención.

Conclusiones: Los adultos con CC reportan una demanda de recursos de salud expresada en controles médicos, consultas de urgencia, hospitalizaciones y procedimientos, que aparentemente no es sólo por factores relacionados a sus patologías. Es necesario elaborar estrategias de atención en salud para responder a las necesidades de esta creciente población y que permitan un uso razonable de recursos. Es perentorio estimular el control periódico dental y la profilaxis de endocarditis.

Correspondencia:

Dr. Fernando Baraona Reyes

División de Enfermedades Cardiovasculares, Facultad de Medicina,

Pontificia Universidad Católica de Chile

fbaraona@med.puc.cl 


\section{Health resources used by adults with congenital heart disease in Chile}

Background: A greater use of health resources by adults with congenital heart disease has been reported in international studies.

Aim: to describe the use of health resources by adults with CHD in Chile including frequency of medical controls, emergency consultations, hospitalization and medical procedures. Dental consultations and prophylaxis for infective endocarditis were also evaluated Methods: a cross sectional analytic study was performed on patients with CHD followed in the Instituto Nacional del Tórax, Santiago, Chile, during 2014 and 2015. Patients were asked to answer a questionnaire and data was analyzed according to gender, and type of CHD (Bethesda classification). Statistics were computed using SPSS.

Results: Valid answers were obtained from 363 sub- jects, 180 males $(49.6 \%)$. Mean age was $20.2 \pm 13.3$ years. 78 patients $(21.5 \%)$ had simple, $161(44.4 \%)$ moderate and 124 (34.2\%) severe CHD. 296 (84.6\%) patients had a medical control within the last year. 151 $(41.6 \%)$ had attended a medical emergency service $\geq$ 1 occasion, 76 (20.9\% had >= 1 hospitalizations and $54(14.9 \%)$ had been subjected to a medical procedure related to their CHD. Women had more medical controls than males $(48,1 \%$ vs $35 \%, \mathrm{p}<0,05) .122(33.6 \%)$ had no dental control in the last year and $45 \%$ of those attending had no prophylaxis for IE

Conclusion: Adults with CHD use health resources including medical visits, hospitalizations and procedures, apparently not all of them related to their CHD. Strategies to optimize use of health resources should be designed. Emphasis on dental controls and prophylaxis for infective endocarditis is needed. 
Introducción: Los avances de la cirugía cardíaca han permitido una mejoría en la sobrevida de los pacientes con cardiopatía congénita (CC). ${ }^{1} \mathrm{El}$ aumento de esta población de adultos junto con la necesidad de corregir la secuelas de los tratamientos efectuados y la aparición de comorbilidades, se ha traducido en una creciente demanda de recursos de salud por parte de este particular grupo de pacientes. Reportes internacionales han demostrado un elevado uso de recursos salud recursos de salud traducido en mayor número hospitalizaciones, frecuentes consultas a servicios de urgencias y procedimientos. ${ }^{2-8}$ No obstante, las intervenciones realizadas en un centro de referencia se justifican por el impacto significativo que tienen en la calidad de vida y sobrevida de estos pacientes ${ }^{3}$.

Se desconoce el número de pacientes adultos con $\mathrm{CC}$ en Chile, pero de acuerdo a reportes internacionales se estima una prevalencia de 3.000 pacientes por 1 millón de habitantes. ${ }^{9}$ En el Instituto Nacional del Tórax (INT), que es el centro de referencia nacional desde el año 2000, se controlan cerca de 2.500 pacientes mayores de 15 años, se realizan 1350 controles médicos anuales e ingresan 16 pacientes nuevos mensualmente (datos no publicados). Por otro lado, el progreso observado con la implementación de la ley GES (Garantías Explícitas en Salud) en pacientes con $\mathrm{CC}$ operables en menores de 15 años desde el año 2006, permite suponer que la población de adultos seguirá creciendo así como la necesidad de consultas médicas, procedimientos hemodinámicos y cirugía cardíaca, constituyéndose en un nuevo desafío de salud pública nacional. Las guías internacionales en el manejo de pacientes con CC recomiendan el control cardiológico periódico en un centro de referencia y por especialistas en el área, con una frecuencia que varía entre 6-12 meses para aquellos pacientes con CC compleja (por ej. ventrículo único) a 12-24 meses para aquellos con CC moderada y que se encuentren estables. Así mismo, se recomienda un control dental regular (al menos anual) junto con profilaxis de endocarditis en aquellos pacientes de riesgo ${ }^{10}$.

Entonces, es importante conocer los requerimientos de recursos de salud de esta particular población para su eficiente utilización en nuestro sistema de salud.

El objetivo de este estudio fue evaluar la demanda de recursos de salud por pacientes adultos con CC en nuestro país y en particular, la frecuencia del control de salud dental junto con la recomendación de profilaxis de endocarditis.

\section{Métodos:}

Se realizó un estudio cuantitativo de corte transversal, analítico e individual en pacientes con CC de distinta complejidad que se controlaron en INT, Santiago, Chile, durante el período Junio 2014 a Junio 2015. El grado de complejidad se definió según la clasificación descrita en el consenso de Bethesda ${ }^{11}$ y se corroboró el diagnóstico referido con la información contenida en la base de datos del centro de CC. Se excluyó a aquellos pacientes que respondieron la encuesta y no fue posible clasificar el grado de complejidad de su CC.

La encuesta fue elaborada por cardiólogos del centro de CC y recolectó variables demográficas, clínicas y uso de recursos de salud. En particular, se exploró la fecha del último control médico, la especialidad de éste, fecha del último control cardiológico, consultas en urgencias, número de hospitalizaciones y número de procedimientos efectuados. En términos de salud oral, se preguntó la fecha del último control dental y si había tomado antibióticos como profilaxis de endocarditis. La forma en que se aplicó este instrumento fue presencial, impresa y entregada a los pacientes mientras esperaban su control cardiológico ambulatorio en INT. Ésta fue contestada de forma autónoma por cada paciente o su acompañante en el caso de aquellos que no estaban capacitados para hacerlo.

Los datos fueron extraídos e ingresados a una base de datos computacional, utilizándose el programa Microsoft Excel 2013. El análisis estadístico se realizó mediante el programa SPSS. Para el análisis de los datos se compararon las respuestas en cada ítem con respecto a grupos separados según sexo en una primera instancia y luego según tipo de CC. Las características basales de los grupos son presentadas en promedios y desviación estándar si corresponde. Las variables categóricas son descritas en porcentajes o números absolutos y comparadas a través del test chi-cuadrado de Pearson. Esta encuesta fue aprobada por el Comité de Ética de INT y todos los pacientes firmaron un consentimiento informado antes de responder la encuesta.

\section{Resultados}

\section{Descripción de la población}

Un total de 372 pacientes respondieron la encuesta y se excluyeron 9 pacientes por inconsistencia de datos. Así, la muestra estuvo constituida por 183 mujeres $(50,4 \%)$ y 180 hombres (49,6\%), con una edad promedio de $29,2 \pm 13,3$ años (rango 15-79 años), siendo las mujeres de mayor edad ( 31,5 vs 27,8 años, p $<0,05)$. Esto también se observó en los pacientes con CC simple vs moderada-compleja: 37,8 vs 27,5 años, $(p<0,05)$. Según el grado de complejidad de la CC, los pacientes se clasificaron en: simple 78 
$(21,5 \%)$, moderada $161(44,4 \%)$ y compleja $124(34,2 \%)$. Menos del $1 \%$ de los pacientes correspondieron al grupo etario de mayores de 65 años y todos ellos eran portadores de una CC simple. La distribución de género fue homogénea en cada grupo y entre los grupos. Con respecto a la distribución por residencia de los pacientes: 50,3\% vivía en el área Metropolitana. Todos los pacientes son beneficiarios del Fondo Nacional de Salud (FONASA). En relación al nivel educacional: $42,5 \%$ reportó escolaridad completa y 21,3\% estaba cursando o completó estudios de educación superior. Con respecto a la situación laboral: $38 \%$ reportó realizar un trabajo remunerado, $10 \%$ refirió estar cesante y 7,4\% jubilado. En relación a estas variables socioeconómicas, no se observó correlación con el tipo de $\mathrm{CC}$, pero se observó un mayor número de pacientes jubilados en el grupo de CC compleja.

\section{Atención de Salud}

Control médico. La mayoría de los pacientes $(81,5 \%)$ asistió a control en el último año y sólo $54(14,9 \%)$ pacientes lo hicieron hace más de 1 año, siendo esto más frecuente en el grupo de los hombres $(19,4 \%$ vs $10,4 \%, \mathrm{p}<0,05)$. No se observó diferencias según tipo de CC. Con respecto a la pregunta por la especialidad de su último control médico, $55,4 \%$ de los pacientes visitó un cardiólogo y de ellos, principalmente fueron hombres $(63,3 \%$ vs $47,5 \%$, $\mathrm{p}<0,05)$. (Figura 1).

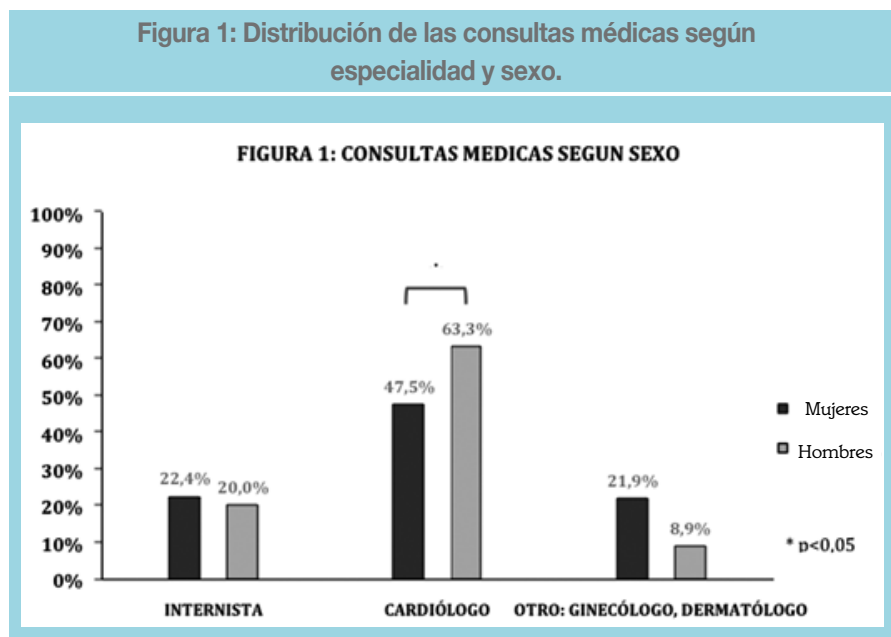

\section{Consulta en el servicio de urgencias}

$151(41,6 \%)$ pacientes asistieron una o más veces al servicio de urgencias en el último año, principalmente mujeres $(48,1 \%$ vs $35 \%, p<0,05)$. No se observó diferencias según el tipo de CC. (Figura 2).
Figura 2: Utilización de recursos de salud representado por consultas a servicios de urgencias (A), hospitalizaciones (B) y procedimientos (C) según tipo de CC.

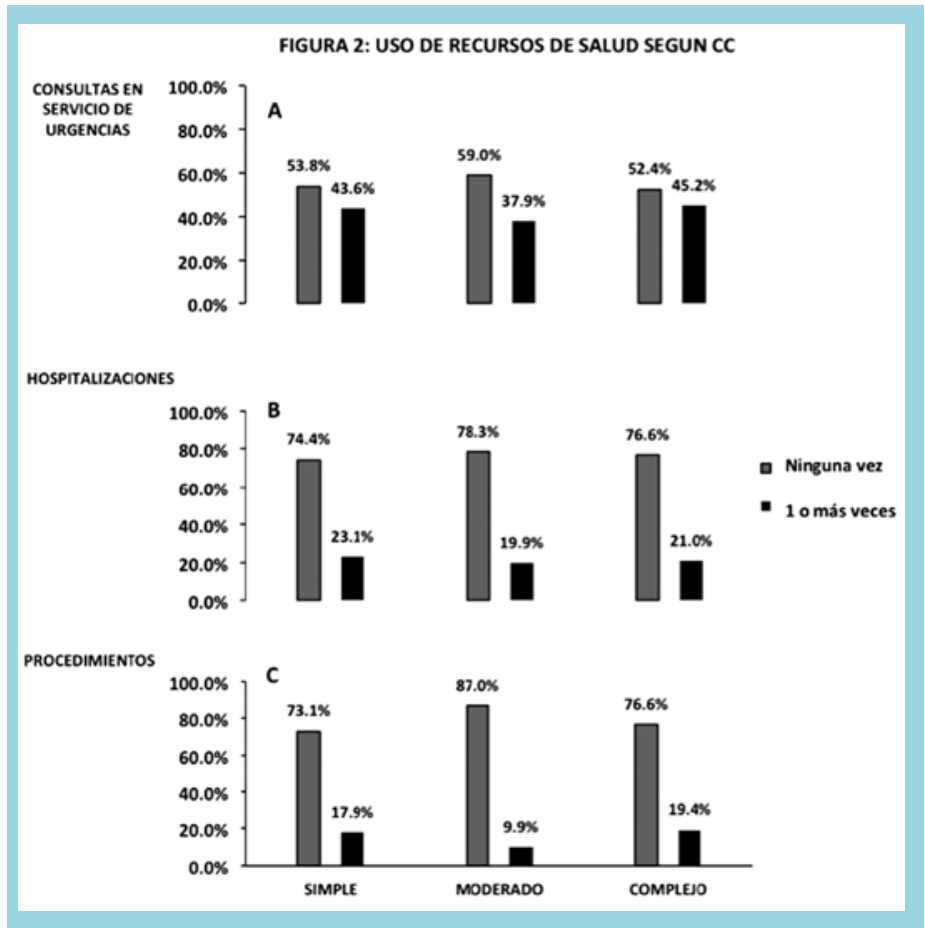

Procedimientos y hospitalizaciones

$14,9 \%$ de los pacientes se ha realizado uno o más procedimientos (sondeos cardíacos y estudios electrofisiológicos) y $20,9 \%$ fue hospitalizado durante el último año. No hubo diferencias significativas en el análisis por sexo o tipo de CC. (Figura 2).

\section{Salud oral}

Control odontológico. $122(33,6 \%)$ pacientes reportaron haber asistido a control dental hace más de 1 año y no se observó diferencias estadísticamente significativas según sexo o tipo de CC. $165(45,5 \%)$ reportó no haber recibido ningún consejo sobre profilaxis antibiótica de endocarditis, sin observar diferencias estadísticamente significativas por sexo o tipo de CC. (Figura 3).

\section{Discusión}

Este es el primer estudio realizado en nuestro país que describe la demanda por recursos de salud de esta particular población. Así, la creación de un sistema integrado por el actual centro de referencia y una futura red nacional de prestadores, pudiera ser una solución para responder a esta creciente demanda y para una mayor eficiencia en el uso de los recursos disponibles. Si bien estos procedimientos son intervenciones de alto costo, los beneficios en térmi- 
Figura 3: Prevalencia del consejo médico sobre profilaxis de endocarditis bacteriana para su control de salud oral.

FIGURA 3: EDUCACION SOBRE PROFILAXIS ENDOCARDITIS SEGUN CC

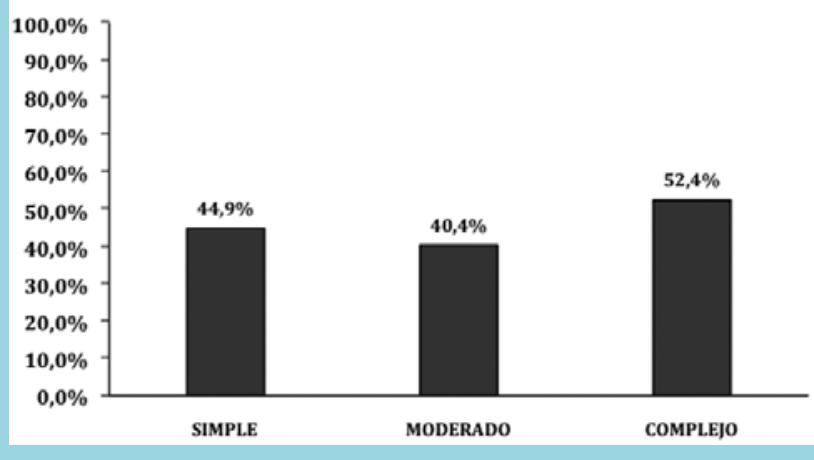

nos de calidad de vida y sobrevida (con la posibilidad de retomar una vida laboral activa), fueron reportados por Hunter et al ${ }^{3}$, justificando su realización.

Uno de los hallazgos interesantes a destacar de este trabajo es que, si bien el centro de CC del INT es el centro de derivación nacional de estas patologías y concentra una mayor proporción de pacientes con CC moderadas-complejas, las necesidades de atención parecen estar más relacionadas al género que al tipo de CC. A diferencia de lo reportado por Mackie et $\mathrm{al}^{5}$, donde se asoció la mayor demanda con la CC compleja. Una hipótesis que explique este fenómeno es el pobre conocimiento de las CC tanto por los pacientes como por los prestadores de salud, lo que se traduciría en consultas frecuentes, exámenes innecesarios y hospitalizaciones injustificadas. Con esta encuesta no es posible conocer los motivos de consulta y/o hospitalizaciones de los pacientes. Tampoco el centro tiene acceso a las fichas clínicas de los lugares de atención. Sería interesante evaluar el impacto de intervenciones como la educación de los pacientes y la creación de redes de derivación de pacientes, en el uso de recursos de salud.

En particular, las mujeres resultaron tener una mayor frecuencia y variedad de consultas médicas (cardiológicas, gineco-obstétricas y otras), así como, más consultas en servicios de urgencia. Esto también ha sido reportado en la población general de nuestro país ${ }^{12}$. Sería interesante explorar si esto responde a razones de origen sociocultural o tiene relación con factores biológicos propios del sexo femenino. Por ejemplo, el antecedente de un embarazo previo en mujeres con CC sería un factor protector al requerir menores recursos de salud (lo que traduciría un mejor estado de salud $)^{13}$.

Se ha postulado que el control de la salud oral debiera formar parte de los cuidados estandarizados de esta po- blación, ${ }^{10}$ por su rol en la prevención de endocarditis bacteriana. Es conocida la mayor incidencia de endocarditis en pacientes con CC en comparación con la población general ${ }^{14}$. Entonces, es notorio que un tercio de la muestra de este estudio que acude a controles regulares en este centro, no haya cumplido con su control dental en el último año y que cerca de la mitad de la muestra no haya recibido profilaxis antibiótica. Lamentablemente, no es posible conocer las causas de este fenómeno mediante esta encuesta. Factores como desconocimiento tanto de los pacientes como de los prestadores de salud, así como la residencia en regiones de un grupo importante de pacientes, son explicaciones plausibles. El uso de metodología cualitativa facilitaría conocer mejor este fenómeno. Si bien la Encuesta Nacional de Salud 2009-2010 ${ }^{15}$ reportó peores resultados en términos de salud oral en la población general, la fragilidad de los pacientes adultos con CC motiva la creación de estrategias de atención preferente, como podría ser la incorporación en las Garantías Explícitas de Salud.

\section{Limitaciones}

Como toda encuesta, la información obtenida se basa en el autoreporte del paciente. Por otro lado, no conocemos el número de encuestas completadas en forma independiente por el paciente. Esto dificulta la interpretación de los datos ya que no hay forma de corroborar la veracidad de las respuestas; sin embargo, es razonable pensar que la encuesta permitió tener una buena percepción del estado de salud en un momento determinado.

Esta encuesta se aplicó a pacientes que asistieron a control cardiológico programado y cuya frecuencia de control es anual. Esto pudiera explicar por qué el control cardiológico resultó ser una atención frecuente. Sería interesante conocer qué pasa con todos aquellos pacientes que no están siendo controlados en este centro, porque abandonaron su cuidado médico y/o de la especialidad durante la transición desde la atención de salud infantil hacia la adulta.

\section{Conclusiones}

Los adultos con CC reportan una demanda de recursos de salud expresada por controles médicos, consultas de urgencia, hospitalizaciones y procedimientos, que aparentemente no son sólo por factores relacionados a sus patologías. Específicamente, el grupo de mujeres cardiópatas pareciera concentrar estos requerimientos. Es perentorio elaborar estrategias de atención en salud para responder a las necesidades de esta creciente población y que permitan un uso razonable de recursos junto con estimular el control periódico dental y la profilaxis de endocarditis. 


\section{Referencias:}

1. WARNES CA, LIBERTHSON R, DANIELSON GK, DORE A, HARRIS L, HOFFMAN JI, et al. Task force 1: the changing profile of congenital heart disease in adult life. J Am Coll Cardiol 2001; 37: 1170-1175.

2. AVILA P, MERCIER LA, DORE A, MARCOTTE F, MONGEON FP, IBRAHIM R, et al. Adult congenital heart disease: a growing epidemic. Can J Cardiol 2014; 30: S410-419.

3. HUNTER RM, ISAAC M, FRIGIOLA A, BLUNDELL D, BROWN K, BULL K. Lifetime costs and outcomes of repair of Tetralogy of Fallot compared to natural progression of the disease: Great Ormond Street Hospital cohort. PLoS One 2013; 8: e59734.

4. LU Y, AGRAWAL G, LIN CW, WILLIAMS RG. Inpatient admissions and costs of congenital heart disease from adolescence to young adulthood. Am Heart J 2014; 168: 948-955.

5. MACKIE AS, PILOTE L, IONESCU-ITTU R, RAHME E, MARELLI AJ. Health care resource utilization in adults with congenital heart disease. Am J Cardiol 2007; 99: 839-843.

6. OPOTOWSKY AR, SIDDIQI O K, WEBB GD. Trends in hospitalizations for adults with congenital heart disease in the U.S. J Am Coll Cardiol 2009; 54: 460-467.

7. TUTAREL O, KEMPNY A, ALONSO-GONZALEZ R, JABBOUR R, LI W, UEBING A, et al. Congenital heart disease beyond the age of 60: emergence of a new population with high resource utilization, high morbidity, and high mortality. Eur Heart J 2014; 35: 725-732.

8. VERHEUGT CL, UITERWAAL CS, VAN DER VELDE ET, MEIJBOOM FJ, PIEPER PG, SIESWERDA GT, et al. The emerging burden of hospital admissions of adults with conge- nital heart disease. Heart 2010; 96: 872-878.

9. VAN DER BOM T, BOUMA BJ, MEIJBOOM FJ, ZWINDERMAN AH, MULDER BJ. The prevalence of adult congenital heart disease, results from a systematic review and evidence based calculation. Am Heart J 2012; 164: 568-575.

10. WARNES CA, WILLIAMS RG, BASHORE TM, CHILD JS, CONNOLLY HM, DEARANI JA, et al. ACC/AHA 2008 Guidelines for the Management of Adults with Congenital Heart Disease: a report of the American College of Cardiology/ American Heart Association Task Force on Practice Guidelines (writing committee to develop guidelines on the management of adults with congenital heart disease). Circulation 2008; 118: e714-833.

11. WEBB GD, WILLIAMS RG. Care of the adult with congenital heart disease: introduction. J Am Coll Cardiol 2001; 37: 1166.

12. VEGA J, BEDREGAL P, JADUE L, DELGADO I. [Gender inequity in the access to health care in Chile]. Rev Med Chil 2003; 131: 669-678.

13. ZOMER AC, IONESCU-ITTU R, VAARTJES I, PILOTE L, MACKIE A S, THERRIEN J, et al. Sex differences in hospital mortality in adults with congenital heart disease: the impact of reproductive health. J Am Coll Cardiol 2013; 62: 58-67.

14. VERHEUGT CL, UITERWAAL CS, VAN DER VELDE ET, MEIJBOOM FJ, PIEPER PG, VEEN G, et al. Turning 18 with congenital heart disease: prediction of infective endocarditis based on a large population. Eur Heart J 2011; 32: 1926-1934.

15. ENCUESTA NACIONAL SALUD CHILE. ENS 20092010. web.minsal.cl/portal/url/item/bcb03d7bc28b64dfe040010165012d23.pdf 